\title{
PHASE CALIBRATION OF MULTIBASELINE SAR DATA BASED ON A MINIMUM ENTROPY CRITERION
}

\author{
M. Pardini, K. Papathanassiou \\ German Aerospace Center (DLR) \\ Microwave and Radar Institute (HR) \\ Oberpfaffenhofen (Germany)
}

\author{
V. Bianco, A. Iodice \\ Università degli Studi di Napoli \\ "Federico II" \\ Naples (Italy)
}

\begin{abstract}
Prior to any processing of multibaseline (MB) synthetic aperture radar (SAR) data stacks, a MB phase calibration is necessary to compensate for phase contributions due to platform motions and/or atmospheric propagation delays. Classical calibration methods rely on the detection of point-like scatterers. However, especially in natural scenarios, their final calibration performance could be impaired by the nature of the scattering and by the typical low number of baselines. In this paper, we propose a calibration method based on the minimization of the entropy of the vertical profile of the backscattered power. This allows to potentially exploit the MB SAR signal independently of the nature of the scattering. The proposed method has been tested by processing simulated and real airborne datasets of a forest stand.
\end{abstract}

Index Terms - Synthetic aperture radar, tomography, calibration, entropy minimization, adaptive beamforming

\section{INTRODUCTION}

It is well demonstrated that the processing of multibaseline (MB) SAR data allows an improved imaging and characterization of the observed scene. Unfortunately, especially in airborne SAR acquisitions, residual non-compensated platform motions result in baseline estimation errors. On the other hand, atmospheric propagation delays have to be accounted in the case of multiple space borne acquisitions. In both cases, the MB data stack is affected by unknown phase contributions different from track to track $[1,2]$. As a consequence, prior to any $\mathrm{MB}$ coherent processing, it is necessary to correct the data stack for these phase residuals [3].

From a signal processing perspective, the phase calibration can be carried out by following two different classes of algorithms. A first class makes use of a grid of targets of opportunity (generally point-like) which remain stable during the entire acquisition time span. MB calibration methods based on the detection of the so-called PS (persistent scatterer) and CS (coherent scatterers) have been experimented in [3] and [4], respectively. However, the effectiveness of this class of techniques is scenario-dependent. In fact, in natural scenarios the presence of PS/CS is more reduced than in urban scenarios. Moreover, as the number of baselines is typically kept low to avoid temporal decorrelation problems, the detection performance worsen and the calibration quality degrades dramatically. As a consequence, the MB phase calibration in presence of a few tracks turns out to be a challenging task. To overcome all these problems, a second class of algorithms has been introduced based on some kind of MB autofocus. With particular reference to forest scenarios, in [5] an approach has been proposed which performs a sort of autofocus on the ground scatterer, after its separation from the canopy scatterer by exploiting a full-pol MB data stack. However, this method could not be effective when calibrating over an area with dense vegetation, where the isolation of the ground scatterer results to be more difficult. A different method has been proposed in [6], which estimates the calibration phases by optimizing an ad-hoc measure of the contrast of the vertical profile of the backscattered power (viz. tomogram) based on the statistics of the profile amplitudes measured on a range-azimuth area around the SAR cell of interest.

This work proposes and investigates a new calibration method based on the minimization of the entropy [7] of the adaptive beamforming $(\mathrm{ABF})$ tomogram in the SAR cell under test. Indeed, in the context of the information theory the minimization of the entropy of a functional with respect to some parameters is equivalent to the maximization of its sharpness, as it get reduced when phase miscalibrations corrupt the data [2]. It is worth noting that the proposed method exploits the whole MB information, and there is no need to focus on a particular target or on a scatterer in the profile. In this way, the spatial coverage of calibration targets is full and a single polarimetric channel is sufficient to correct properly the whole MB data stack. Moreover, the proposed technique can be considered alternative to the one in [6], with the advantage that the minimum entropy-based solution can operate with a single cell, thus overcoming possible performance limitations due to profile variability in the range-azimuth plane.

\section{ENTROPY MINIMIZATION}

Let $\{\mathbf{y}(n)\}_{n=1}^{N}$ be the $K$-dimensional MB complex data vectors collected in $N$ adjacent range-azimuth pixels composing the multilook cell under test, being $K$ the number of images in the data stack. Assuming the phase errors very correlated in the range-azimuth plane $[3,5]$, for each pixel it results:

$$
\mathbf{y}(n)=\mathbf{y}_{0}(n) \odot \exp \{j \phi\}, n=1, \ldots, N,
$$

where $\left\{\mathbf{y}_{0}(n)\right\}_{n=1}^{N}$ are the perfectly calibrated MB data vectors, $\phi$ is a $K$-dimensional vector containing the unknown residual miscalibration phases $\phi_{k}$ with respect to the master image, and " $\odot$ " denotes the Hadamard product. Given $\{\mathbf{y}(n)\}_{n=1}^{N}$, it is possible to estimate the $\mathrm{ABF}$ vertical profile $f(z)$. It is well-known that $f(z)$ show remarkable height super-resolution, sidelobe rejection and sharpness in absence of miscalibration $(\phi=0)$. However, when $\phi$ increases in magnitude, $f(z)$ will look, in general, less sharp with inflated sidelobes and possible mislocations in height of the imaged scatterers. In the information theory, the profile sharpness is expressed by resorting to the concept of entropy. In particular, the Renyi entropy 
[7, 8] is a function of $f(z)$ in the height interval of interest as follows:

$$
S_{r}[f(z)]=\frac{1}{1-r} \ln \sum_{m=1}^{M} f^{2 r}\left(z_{m}\right)-\frac{r}{1-r} \ln \sum_{m=1}^{M} f^{2}\left(z_{m}\right),
$$

where $z_{m}$ are the heights at which the the profile is sampled. When $r=2$, the Renyi entropy can be used to measure the sharpness of the vertical profile. Indeed, the higher $S_{2}[f(z)]$, the higher the entropy, and the lower the sharpness. Consequently, the vector $\phi$ can be estimated as follows:

$$
\hat{\phi}=\arg \min _{\phi} S_{2}[f(z, \phi)],
$$

in which the dependence of $f(z)$ on $\phi$ has been explicated for the sake of clarity. It is worth noting that the minimization of the entropy will introduce an unknown height shift in the profile. This is due to the fact that the height interval of interest is typically much narrower compared with the non-ambiguous range in height. For this reason, in order to retrieve the correct position in height of the vertical profiles, the following procedure in 5 steps is proposed:

1. Calculate the interferogram at each baseline with respect to the master image. The interferometric phase at the $k$-th interferogram can be written as:

$$
\psi_{k}=k_{z, k} z_{0}+\delta_{k}+\phi_{k}, k=1, \ldots, K-1
$$

where $z_{0}$ is the DEM height and $\delta_{k}$ is the DEM error variable from baseline to baseline;

2. Compensate the phases in (4) in the MB signal (1), obtaining:

$$
\mathbf{y}_{C}(n)=\tilde{\mathbf{y}}_{0}(n) \odot \exp \{-j \boldsymbol{\delta}\},
$$

where $\tilde{\mathbf{y}}_{0}(n)$ is a height-shifted version of $\mathbf{y}_{0}(n)$ of a quantity $z_{0}$, and $\boldsymbol{\delta}$ contains the baseline-dependent DEM errors $\delta_{k}$;

3. The phases $\delta$ can be be estimated by applying the optimization (3) to $\left\{\mathbf{y}_{C}(n)\right\}_{n=1}^{N}$;

4. The estimated phases $\delta$ can be compensated in (4). The remaining MB phase set can be used to estimate $z_{0}$ and $\phi$ by exploiting the low variability of $\phi$ in the range-azimuth plane. Starting e.g. from a stable non-forest pixel or a corner reflector, a recursive procedure can be set up compensating the error $\phi$ in the cell under analysis by using the phases $\phi$ already obtained in an adjacent cell, thus allowing to estimate $z_{0}$ in presence of a very low phase disturbance.

5. Finally, the calibrated data vector is obtained by shifting the vector $\tilde{\mathbf{y}}_{0}(n)$ obtained at Step 3 of a quantity $-z_{0}$.

It is worth remarking the importance of Step 2. Indeed, the compensation of the interferograms $(i)$ reduces the calibration problem to a better conditioned one, i.e. with phase errors with a lower magnitude; (ii) allows to compensate for the height shifts introduced by the entropy minimization; (iii) from the point of view of the implementation, it makes easier the choice of a height interval of interest in which the scattering is supposed to be included. As concerns the minimization of the entropy functional in Step 3, a closed form solution of (3) does not exist, thus a numerical procedure must be adopted. $S_{2}[f(z)]$ a non-linear, non-convex, $(K-1)$-dimensional functional. Here, the minimization has been tackled by means of a Coordinate Descent procedure [8], in which the entropy functional is minimized with respect to a single parameter at time while holding the others fixed.

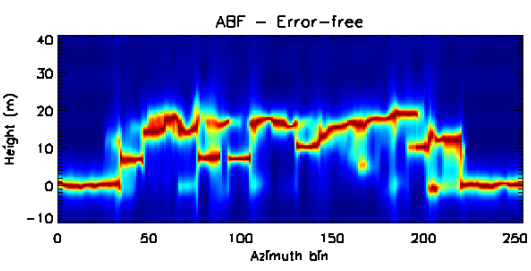

(a) $\phi=0$

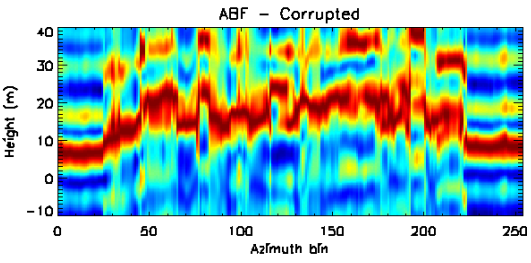

(b) $\phi \neq 0$

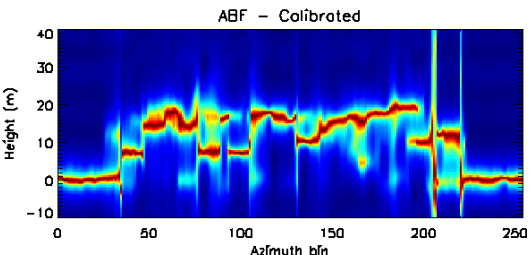

(c) Calibrated with ME

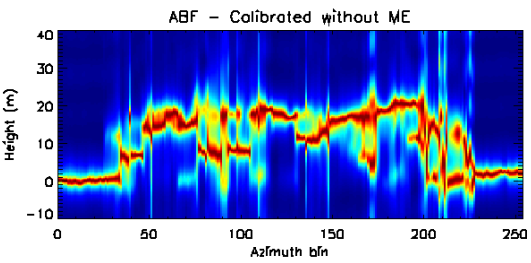

(d) Calibrated without ME

Fig. 1. Simulated tomo slices.

\section{EXPERIMENTS WITH SIMULATED DATA}

The performance of the minimum entropy-based (ME) calibration method have been quantified by processing a simulated L-band MB data set obtained with the PolSARproSim software ${ }^{1}$. The acquisition parameters have been set according to the typical values of the DLR's E-SAR platform. The complete stack consists of 5 images with baselines $5 \mathrm{~m}, 10 \mathrm{~m}, 15 \mathrm{~m}, 25 \mathrm{~m}$, as in the TempoSAR'08 dataset used for the experiments with real data (see next Section). The simulated area is a forest stand (mean tree height of $20 \mathrm{~m}$ ) on a flat ground located at zero height. The simulated data stack is perfectly calibrated. In the experiments, the miscalibration phases have been inserted according to model (1) as estimated from real data by the CS technique [4].

The $\mathrm{ABF}$ tomogram obtained at a fixed range coordinate are shown in Fig. 1. In absence of phase errors [Fig. 1(a)] it is possible to clearly distinguish the bare soil area from the vegetated one. Profiles are sharp and different scattering layers are resolved in height. If the ABF filter receives in input the phase corrupted data [Fig. 1(b)],

${ }^{1}$ The PolSARproSim software is freely available in the educational software PolSARpro distributed by ESA under the GNU licence. 


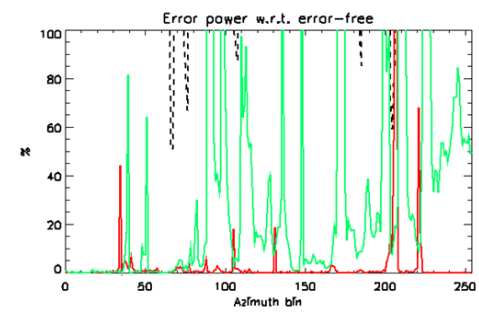

(a) 5 tracks

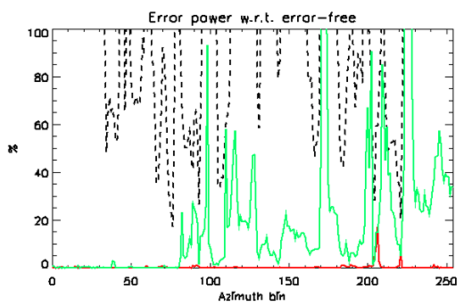

(b) 3 tracks

Fig. 2. Performance obtained in terms of relative reconstruction error. Black-dashed line: corrupted data; green line: reconstructed data without ME; red line: reconstructed data with ME.

inflated sidelobes and a loss of resolution are apparent in the resulting tomogram. A height shift can also occur cell by cell. The overall results of the ME calibration chain (Steps 1-5) applied to the corrupted data is in Fig. 1(c). The proposed ME calibration chain can reconstruct properly the vertical profiles. With the exception of some azimuth coordinates, the obtained profiles look very similar, if not identical, to the error-free ones of Fig. 1(a). In order to emphasize the importance of Step 3 (the minimization of the entropy), the tomogram obtainable avoiding Step 3 is reported as well in Fig. 1(d). It is apparent that Step 3 is of crucial importance.

To quantify the reconstruction performance, we calculated the energy of the profile reconstruction error with respect to the energy of the error-free profile. From the plots of Fig. 2(a), the ME-based calibration can reach a reconstruction error lower than the 5\%, most of the times even better than the $1 \%$. As already noticed, the error is still very high in a very few azimuth bins. It has been verified that a simple way to eliminate those points is a local filtering of the estimated $\phi$. The reconstruction error has been calulated also avoiding Step 3, confirming again that minimization of the profile entropy is crucial. Finally, a 3-track data set extract from the previous one has been processed (baselines $0 \mathrm{~m}, 5 \mathrm{~m}, 15 \mathrm{~m}$ ). The obtained relative errors are reported in Fig. 2(b), showing again the very good reconstruction capability of ME.

\section{EXPERIMENTS WITH REAL DATA}

An E-SAR L-band dataset acquired by DLR in the TempoSAR 2008 campaign over the forest stand of Traunstein (Germany) has been processed. The nominal baseline configuration is like the one used in the simulated experiments. Calibration results are shown with respect to a range line at fixed azimuth and in $\mathrm{HH}$ polarization. In Fig. 3 (top panel) the ABF tomogram obtained without calibration is shown. The effect of the phase errors are apparent, with ambiguities and inflated sidelobes. In the mid panel, the slice obtained after CS calibration [4] is reported. Surprisingly, the CS method performs well already with 5 tracks, allowing to distinguish again between the
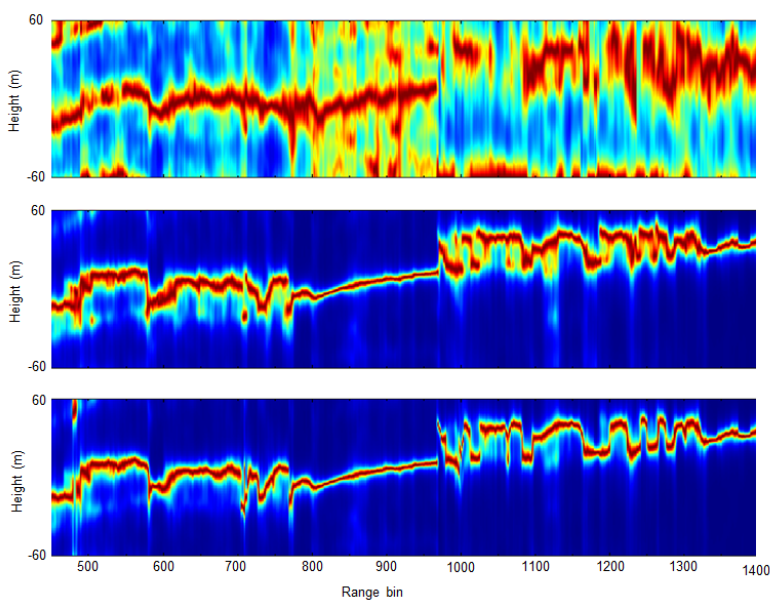

Fig. 3. Tomo slices extracted from the TempoSAR' 08 dataset (5 tracks) with no calibration (top), calibrated with CS (mid), calibrated with ME (bottom).

ground and the vegetation layers. The height resolution capability is recovered and sidelobes rejected. Finally, the bottom panel shows the ME calibrated slice. With respect to the CS-calibrated slices, some small differences can be observed especially in the variation of the radiometric relative levels between the ground and the canopy in most of the forested areas.

As a further experiment, each polarization has been processed independently, and the ME calibration phases have been compared. For each baseline, a strong correlation exists between the phases estimated from the different polarimetric channels (correlation coefficient higher than 85\%). This indicates that the estimated phase errors are the ones coming from uncompensated platform motions, which are not affected by phase information related to scattering in different polarizations, as wanted.

For a better characterization of the calibration performance, some meaningful typical profiles are reported in Fig. 4. In Fig. 4(a) the $\mathrm{ABF}$ profiles in a ground cell are reported. An improvement is visible in terms of height resolution. In fact, the $-3 \mathrm{~dB}$ mainlobe width measures $5.3 \mathrm{~m}$ in the ME profile against the $6.4 \mathrm{~m}$ in the CS profile. The ABF profiles in Fig. 4(b) refer to a forested cell, with spectral peaks at the ground (about $15 \mathrm{~m}$ ) and canopy heights. In this case, after the ME calibration the two peaks are better resolved than in the CS case. Interestingly, a sidelobe close to the ground peak is well suppressed after ME calibration, with a gain of $5 \mathrm{~dB}$ in sidelobe rejection with respect to the CS profile. Analyses have been carried out also to assess the gain in terms of radiometric fidelity. Fig. 4(c) show the CS and ME ABF profiles in a forested cell. Apparently, the power of the ground (at about $-10 \mathrm{~m}$ ) relative to the one of the canopy changes noticeably from CS to ME. In order to assess which is the right ground-to-volume power relation, an error-free profile has been simulated in such a way that the Fourier-based spectrum (highly insensitive to residual phase errors) of the simulated profile matches with the one estimated from the real data. In Fig. 4(d) the simulated $\mathrm{ABF}$ profile is compared with the ME one. A good agreement between the two profiles can be observed. Since the simulated one has been generated in absence of errors, such an agreement is an indication that the proposed ME-based method provides better calibration phases than the CS.

In the last experiment, we considered a dual baseline acquisition 


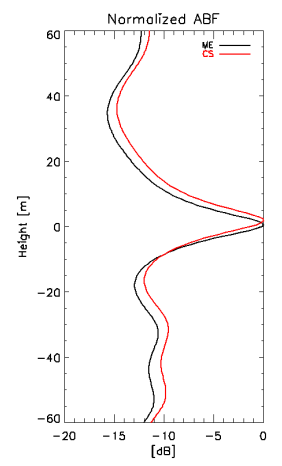

(a)

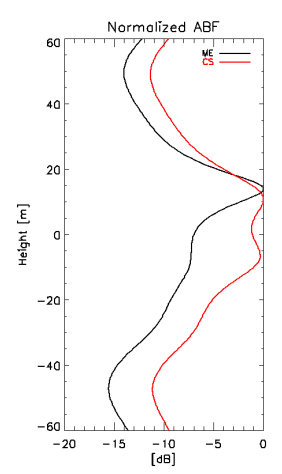

(c)

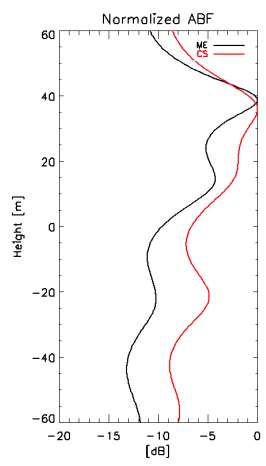

(b)

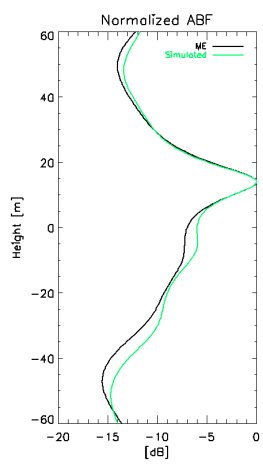

(d)
Fig. 4. Examples of profiles.

by processing the images in the data stack with baselines $5 \mathrm{~m}$ and $15 \mathrm{~m}$. Fig. 5 reports a very first-cut result, in which the CS method is not able to estimate the calibration phases, as the quality of the retrieved spectrum is very low. While this method fails (at least in this azimuth coordinate depending on the sparsity of the detected CS), the proposed ME can retrieve a tomographic slice with very reduced ambiguities and with improved sidelobe rejection. Some residual lobes in near-range are still present, which are actually due to the very poor PSF in height. Experiments are ongoing in further assessing the goodness of the reconstruction.

\section{CONCLUSIONS}

The proposed ME-based calibration method has been tested with simulated and real data. The tests with simulated data have demonstrated the capability of ME in reconstructing a profile with an error typically between the $1 \%$ and the $5 \%$ even with 3 tracks only. On the other hand, the real data results have given indications about the superiority of the ME-based calibration with respect to the classical methods based on the detection of point like targets, at the cost of an increased computational load. From the experiments, the proposed method turns out to be attractive as it can restore the tomographic radiometric fidelity, which is fundamental when dealing with forest scenarios, and can operate even with only 3 tracks. Nevertheless, experiments will be carried out in order to further assess the reconstruction performance and the applicability of the ME calibration scheme to different kind of scenarios. Further investigations are also needed to characterize the reconstruction performance as a function of the imaged profile.
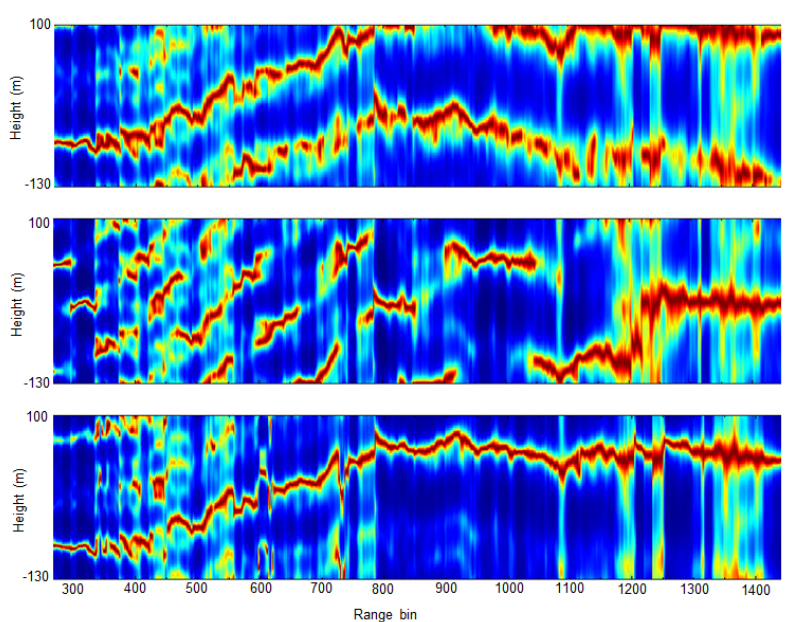

Fig. 5. Tomo slices extracted from the TempoSAR' 08 dataset (3 tracks) with no calibration (top), calibrated with CS (mid), calibrated with ME (bottom).

\section{REFERENCES}

[1] A. Reigber, A. Moreira, "First Demonstration of Airborne SAR Tomography Using Multibaseline L-band Data," IEEE Trans. on Geoscience and Remote Sensing, Vol. 38, No. 5, pp. 21422152, May 2000.

[2] A. Jakobsson, F. Gini, F. Lombardini, "Robust estimation of Radar Reflectivities in Multibaseline InSAR," IEEE Trans. on Aerospace and Electronic Systems, Vol. 41, No. 2, pp. 751-758, Apr. 2005.

[3] S. Tebaldini, A. Monti Guarnieri, "On the Role of Phase Stability in SAR Multibaseline Applications," IEEE Trans. on Geoscience and Remote Sensing, Vol. 48, No. 7, pp. 2953-2966, Jul. 2010.

[4] K. Iribe, K. Papathanassiou, I. Hajnsek, M. Sato, Y. Yokota, "Coherent Scatterer in Forest Environment: Detection, Properties and its Applications," Proc. of IEEE International Geoscience and Remote Sensing Symposium (IGARSS), Honolulu, Hawaii (USA), Jul. 2010.

[5] G. Gatti, S. Tebaldini, M. Mariotti D'Alessandro, F. Rocca, "ALGAE: A Fast Algebraic Estimation of Interferogram Phase Offsets in Space-Varying Geometries," IEEE Trans. on Geoscience and Remote Sensing, Vol. 49, No. 6, pp. 2343-2353, Jun. 2011.

[6] Y. Huang, L. Ferro-Famil, F. Lombardini, "Improved Tomographic SAR Focusing Using Automatic Baseline Error Compensation," Proc. Of ESA PolInSAR Workshop, Frascati, Italy, January 2011.

[7] R. L. Morrison, M. N. Do, D. C. Munson, "SAR Image Autofocus By Sharpness Optimization: A Theoretical Study," IEEE Trans. on Image Processing, Vol. 16, No. 9, pp. 2309-2321, Sep. 2007.

[8] T. J. Kragh, "Monotonic Iterative Algorithm for MinimumEntropy Autofocus," Proc. of 14th Adaptive Sensor Array Processing Workshop (ASAP), Lexington, MA (USA), Jun. 2006. 